\title{
Tracker controls development and control architecture for the Hobby-Eberly Telescope Wide Field Upgrade
}

\author{
Jason R. Mock*a, Joe Beno ${ }^{\mathrm{a}}$, Tom H. Rafferty ${ }^{\mathrm{b}}$, Mark E. Cornell ${ }^{\mathrm{b}}$ \\ ${ }^{a}$ Center for Electromechanics, The University of Texas, 1 University Station R7000, \\ Austin, TX, USA 78712; \\ ${ }^{\mathrm{b}}$ McDonald Observatory, The University of Texas, 1 University Station C1402, Austin, TX, \\ USA 78712
}

\begin{abstract}
To enable the Hobby-Eberly Telescope Wide Field Upgrade, the University of Texas Center for Electromechanics and McDonald Observatory are developing a precision tracker system - a 15,000 kg robot to position a 3,100 kg payload within 10 microns of a desired dynamic track. Performance requirements to meet science needs and safety requirements that emerged from detailed Failure Modes and Effects Analysis resulted in a system of 14 precision controlled actuators and 100 additional analog and digital devices (primarily sensors and safety limit switches). This level of system complexity and emphasis on fail-safe operation is typical of large modern telescopes and numerous industrial applications. Due to this complexity, demanding accuracy requirements, and stringent safety requirements, a highly versatile and easily configurable centralized control system that easily links with modeling and simulation tools during the hardware and software design process was deemed essential. The Matlab/Simulink simulation environment, coupled with dSPACE controller hardware, was selected for controls development and realization. The dSPACE real-time operating system collects sensor information; motor commands are transmitted over a PROFIBUS network to servo amplifiers and drive motor status is received over the same network. Custom designed position feedback loops, supplemented by feed forward force commands for enhanced performance, and algorithms to accommodate self-locking gearboxes (for safety), reside in dSPACE. To interface the dSPACE controller directly to absolute Heidenhain sensors with EnDat 2.2 protocol, a custom communication board was developed. This paper covers details of software and hardware, design choices and analysis, and supporting simulations (primarily Simulink).
\end{abstract}

Keywords: Hobby-Eberly, HET, HETDEX, precision motion control, tracking, robotic motion, dSPACE

\section{INTRODUCTION}

The HET, Figure 1, is the largest telescope at The University of Texas' McDonald Observatory located on Mt. Fowlkes in the Davis Mountain Range in West Texas. A joint venture with The Pennsylvania State University, Stanford University, Ludwig-Maximilians-Universität München, and Georg-August-Universität Göttingen, the HET has been conducting science operations since October, $1999^{1}$. The HET support structure and primary mirror sits at a fixed zenith angle of $35^{\circ}$ and can move in azimuth to access approximately $70 \%$ of the visible sky. The primary mirror, constructed of 91 identical hexagonal segments, forms an $11 \mathrm{~m}$ hexagonal-shaped spherical mirror with a 26,164 mm radius of curvature. The tracker is mounted above the primary mirror on the upper-most portion of the telescope, termed the upper hexagon or "upper hex" for short. The corrector optics mount to the tracker and are positioned via two linear drive systems and a six degree of freedom (DOF) hexapod maintaining the instruments' optical axis normal to and on the focal sphere of the primary mirror.

The Hobby-Eberly Telescope (HET) is currently undergoing a major redesign effort in preparation for the Dark Energy Experiment $^{2,3}$. The upgrade, referred to as HETDEX, involves replacing the current star tracker ${ }^{4}$ along with its drive systems. The catalyst for the replacement of this hardware is the wide field upgrade to the corrector optics. Replacing the current spherical aberration corrector (SAC) with the wide field corrector ${ }^{5}$ (WFC) will increase the HET field of view from 4' to $22^{\prime}$. In addition, the current science instruments ${ }^{6}$ are not in general being replaced. HRS and MRS will 
be re-installed as is. LRS will be replaced with a new instrument, LRS2. VIRUS (Visible Integral-field Replicable Unit Spectrograph ${ }^{7,8}$ ) is being added to the current suite of instruments. These changes will allow the telescope to conduct the largest survey of distant galaxies ever attempted.
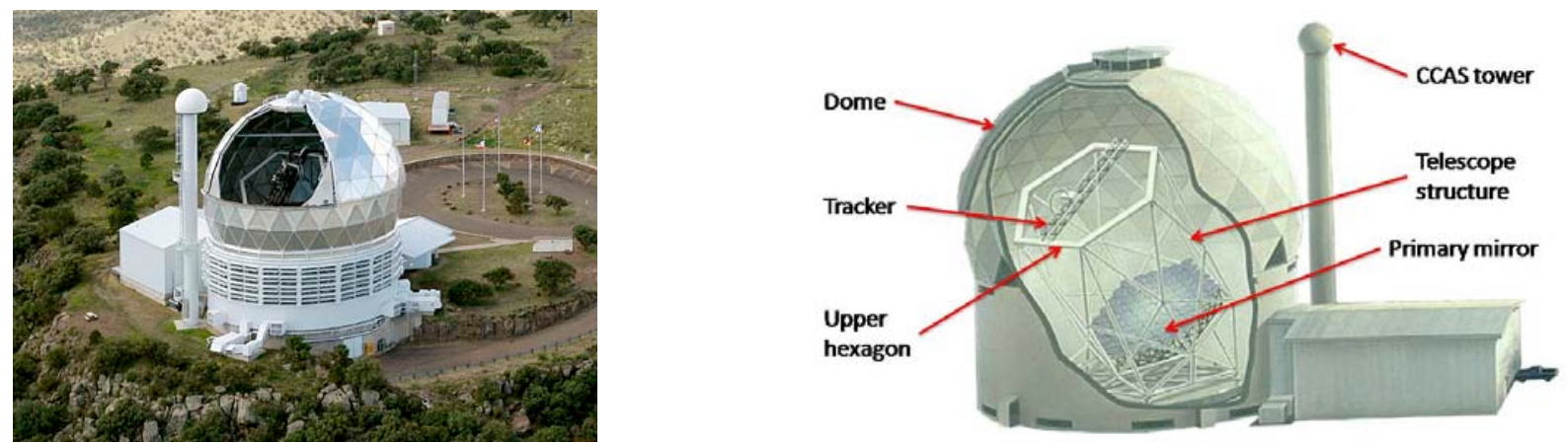

Figure 1. The figure on the left shows an aerial view of the HET with the shutter open. The computer rendering on the right reveals the major components of the telescope.

The primary impact to the HETDEX tracker caused by the replacement of the corrector optics is the approximately seven fold increase in mass of the instruments and supporting hardware. As a result, the HETDEX tracker mass increased on the order of about 5 times that of the HET tracker.

\section{DRIVE SYSTEM}

The HET has two primary linear axes, $\mathrm{X}$ and $\mathrm{Y}$, as defined in Figure 2. The main structural component of the tracker is the bridge. The bridge traverses the upper hex along the $\mathrm{X}$-axis and the corrector optics travel the length of the bridge in the $\mathrm{Y}$-axis. The hexapod provides limited travel in the $\mathrm{X}, \mathrm{Y}$ and $\mathrm{W}$ axes, along with rotations about the $\mathrm{X}(\theta), \mathrm{Y}(\varphi)$ and $\mathrm{W}(\rho)$ axes. The $\mathrm{W}$-axis is the WFC optical axis which tips and tilts to remain on the spherical focal surface of the primary mirror. When the tracker is centered over the primary mirror, the $\mathrm{W}$ and $\mathrm{Z}$ axes are coincident $(\mathrm{X}, \mathrm{Y}$ and $\mathrm{Z}$ axes are orthogonal to each other).

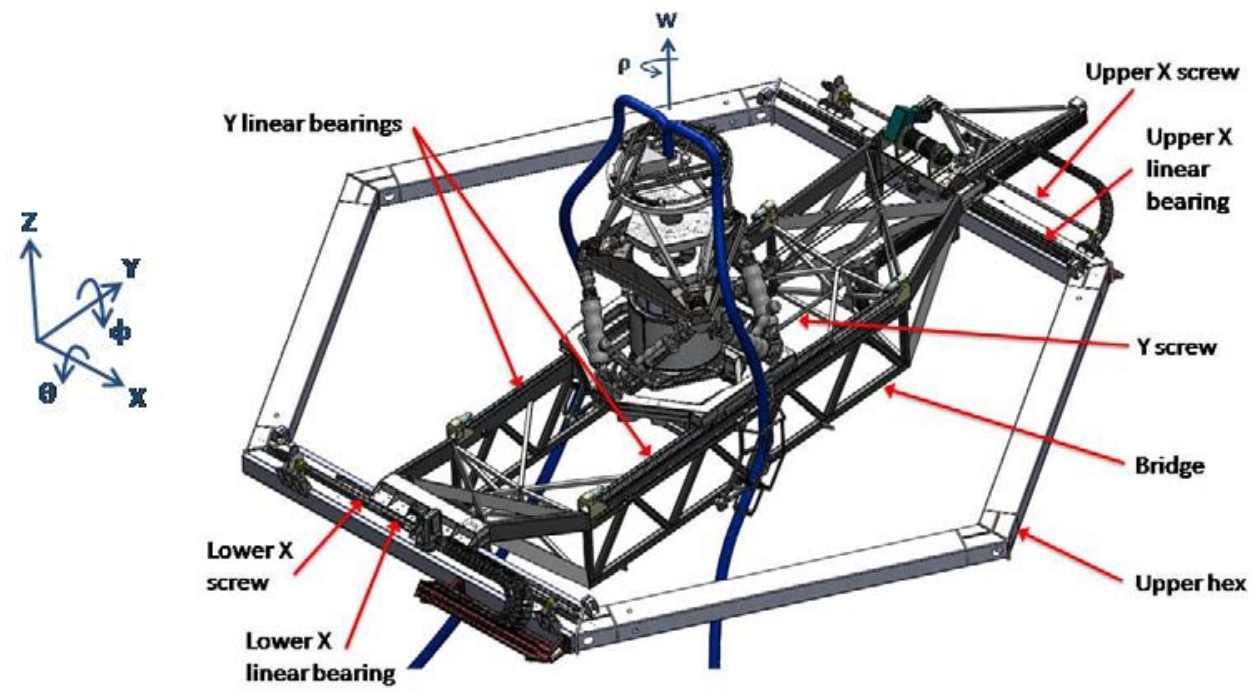

Figure 2. CAD model of the HETDEX tracker showing the key elements of the $\mathrm{X}$ and $\mathrm{Y}$ drive systems and the reference coordinate system 


\subsection{X-Y Drives}

As with the HET, the HETDEX tracker utilizes satellite roller screws to effect tracker movements along the $\mathrm{X}$ and $\mathrm{Y}$ axes. As shown in Figure 2, the $X$ drive consists of a pair of rollers screws situated adjacent to respective linear bearings at the UX and LX beams. The pair of roller screws is driven in unison to move the bridge in the X direction.

Each of the $\mathrm{X}$ drive roller screws is coupled to dual drives, a track drive and a slew drive. The track drive is coupled directly to one end of the screw, referred to as the drive end, and rotates the screw. The slew drive is an offset belt drive that rotates the nut. The slew drive also includes components to couple the nut to the trolleys. This dual drive arrangement is essential to allow slow, precise tracking movements by directly turning the screw and faster slewing movements by rotating the nut. A single motor drive could not be identified which could effectively operate under these two different conditions. Separating the functions allows each drive motor to be selected specifically for a narrow operating window.

\subsection{Hexapod Drive}

A six axis hexapod is an integral part of the Hobby Eberly Telescope (HET) positioning system ${ }^{11}$. In the HET telescope design, the hexapod is used to locate the Primary Focus Instrument Package (PFIP) optical hardware to the appropriate location relative to the primary mirror. The hexapod actuators essentially serve at the mechanical connection between the tracker bridge (x-y motion) and the stiff strongback, which in turn, supports the PFIP optical hardware. The actuators have been designed to meet a broad number of performance requirements, including overall length, stiffness, end joint rotation, heat generation, slewing and tracking speed, and most importantly, positioning accuracy. A positioning accuracy of $+/-0.002 \mathrm{~mm}$ has been demonstrated during the early phases of laboratory testing with the prototype actuator. The primary components in the actuator design are a servo motor, connected to a 60:1 gearbox, and high precision roller screw ${ }^{11}$. The actuators were designed and built by ADS International (Valmadrera, Italy). In addition to performance requirements, the hexapod operation has been heavily scrutinized with regard to personnel and hardware safety and is equipped with limit switches and hard stops, that when engaged will not damage internal actuator components. Using modern modeling and computational techniques ${ }^{12}$, the hexapod actuators were designed to provide the maximum hexapod operational envelope, while preventing unintended impact to adjacent hardware. This iterative design/modeling process allowed the design team to include an adequate amount of excess travel for uncertainties in the existing telescope structure while meeting all of the science requirements.

\subsection{Rho Drive}

The rho stage assembly is mounted on top of the PFIP structure and provides the rotation about the W axis of local telescope coordinate system. The rho stage is supported by a platform that was specifically designed to limit deflection (high stiffness) over all operational positions of the PFIP. A drum is mounted to the platform using three equally spaced curved linear bearings (manufactured by THK). The drum supports the $1000 \mathrm{~kg}$ focal surface hardware through a kinematic mount interface. Included in the focal surface hardware are the integral field units (IFU) fibers which feed reflected light to the VIRUS instruments ${ }^{8}$. The rho stage drum drive system consists of a servo motor connected to a low back-lash gearbox, with a capstan on the output shaft. A set of wire ropes wrap around the drive capstan and connect to opposite end of the drum; one end fixed, the other end pre-loaded with springs. Rho stage angular position will measured using a magnetic tape mounted directly to the outer radius of the drum. The capstan, wire rope, and drum uses the same operating principles as the halyard and winch arrangement for the main sail on a sailboat. The rho stage has an operational envelope of $+/$ - 22 degrees, beyond which a series of mechanical switches and hard stops are engaged. With final hardware currently under construction, the rho stage has slewing and tracking speeds of 1 degree/second and 15 arc-sec/sec, respectively.

\subsection{Constant Force Drive}

The topology of the constant force drive ${ }^{10}$ is similar to a system first deployed on the Southern African Large Telescope [SALT]. A cable hoist anchored to upper end of the bridge connects to the payload with steel wire ropes, and applies constant tension in the uphill direction to offset the Y-axis vector of the payload weight. It is effectively an active electromechanical counterweight system working in parallel with the Y-axis screw-drive. The constant force drive is on a separate dedicated control loop which commands force in proportion to feedback from a load cell, whereas the Y-axis drive controls position as commanded by tracker control system. A spring damper element between the payload and load 
cell/wire rope provides isolation and smoothes response characteristics. This system adds an important safety capability because it does not allow the Y-axis to 'fall' downhill in the event of a Y control fault.

\section{SENSORS}

A large system of sensors is necessary to measure and control the fourteen axes. These sensors are necessary for the precision motion control and health monitoring of the tracker system.

\subsection{X-Y Sensors}

The two $\mathrm{X}$ and one $\mathrm{Y}$ drive axes have identical sensor suites:

- (1) LB382 incremental encoders from Heidenhain corporation. These sensors have a measuring length of 4440 $\mathrm{mm}$, a grating period of 40 microns, and an accuracy grade of $+/-5$ microns.

- (1) PT5DC string potentiometer from Celesco corporation. These sensors have a measuring length of 5 meters and a resolution of $0.2 \mathrm{~mm}$.

- (4) GLC limit switches from Honeywell corporation. Two of these switches are installed at each end of the axis rail.

- (2) AM1 proximity sensors from Automation Direct measure the torque limiter status

- (2) SA2F temperature sensors from Omega measures motor case temperature

Due to the incremental nature of the LB382 sensors they must move slightly on power-up, roughly $25 \mathrm{~mm}$, to find a 'home' mark. During this time the controller has no absolute indication of location. This could result in running into end stops and is generally an undesirable condition. Therefore, a secondary absolute sensor (PT5DC) was chosen to run in parallel. This coarse sensor allows the controller to know the location to within a reasonable precision. This information is used to guide the controller to safely command the system to move enough to find a 'home' mark for the LB382 sensors. Heidenhain corporation does offer an absolute linear sensor that has enough travel capability. The LC183 sensor is offered in measuring lengths up to $4240 \mathrm{~mm}$. Unfortunately, after technical discussions with Heidenhain representatives it was decided that the X rails were not flat enough nor would they be stiff enough to mount the LC183. Therefore the two sensor approach was adopted.

\subsection{Hexapod Sensors}

Each hexapod actuator contains several sensors:

- (1) EQN437 absolute encoder from Heidenhain corporation

- (2)MYCOM F100/80 precision limit switches from Baumer Electric

- (1) SA2F temperature sensor from Omega measures motor case temperature

Length measurement of the ADS actuator is performed using a rotary encoder mounted to the end of the screw. Given the known screw pitch and a well defined screw, very accurate length measurement is possible. The baseline rotary sensor was an EQN425 from Heidenhain corporation. This sensor has both incremental and absolute feedback. The relatively coarse, 13 bits per revolution, absolute information is used only to initially zero the controller. Then the higher resolution incremental feedback is used for control. It was decided to simplify the control and switch to an EQN437 which has only absolute feedback. The EQN 437 outputs 25 bits per revolution and can distinguish 12 bits of revolutions. This sensor returns sufficient resolution for position control and does not require the controller to perform any mode switching between feedback signals.

\subsection{Rho Sensors}

The Rho stage contains several sensors:

- (1) MSK1000 incremental encoder from SIKO 
- (1) EQN437 absolute encoder from Heidenhain corporation

- (3) AM1 proximity sensors from Automation Direct are used as limit switches

- (1) SA2F temperature sensor from Omega measures motor case temperature

Previously, HET used an exposed optical sensor from Heidenhain to read Rho rotation. However, this configuration reacted poorly to dust and debris. Therefore, a magnetic based sensor, which is insensitive to dust and debris, was chosen to read rho position. Due to the incremental nature of the MSK1000 sensor, it must move slightly on power-up to find a 'home' mark. During this time the controller has no absolute indication of location. This could result in running into end stops and is generally an undesirable condition. Therefore, a secondary absolute sensor was chosen to run in parallel. A Heidenhain EQN437 is mounted to the capstan shaft to obtain absolute position. Although the EQN437 will return a very high resolution rotary position, it cannot be relied on as the primary sensor because it is coupled to the Rho drum through a set of cables.

\subsection{Secondary Sensors}

Many other sensors are installed on the tracker for health monitoring and fault detection:

- Coolant temperature

- Coolant flow rate

- Coolant pressure

- Skew limit switches

- $\mathrm{X}-\mathrm{Y}$ brake pressure switches

- Accelerometers

- Inclinometer

- Spring pack load cell

- IFU (Integral Field Unit) load cells

The coolant system on the tracker has many parallel paths with individual flow rate sensors on each. Coolant pressure and temperature are monitored at the main inlet and outlet onto the tracker. Two limit switches detect a skew condition at the top and bottom of the bridge. Pressure switches monitor the brakes on the $\mathrm{X}-\mathrm{Y}$ drives. Several tri-axial accelerometers monitor the carriage and payload for any vibration. A bi-axial inclinometer is installed on the payload to monitor tip and tilt. This sensor will be used for fault detection and not science. Load cells are installed into the IFU bundles to check for snagging issues. A load cell is also installed in the upper spring pack assembly to assure that system maintains the correct load level.

\section{FAULT DETECTION}

Fault detection on the tracker system requires both sensors and software monitoring. Sensors such as limit switches indicate faults directly. Fault detection software monitors analog sensors and compares them to levels that indicate a fault.

\subsection{X-Y Travel Limits}

Each $\mathrm{X}$ or $\mathrm{Y}$ drive has two mechanical limit switches near the end of travel on each side. In addition to the two mechanical limit switches, there is a software limit. These limits operate in sequence and have different purposes:

- Software Limit: Within the controller software, a travel limit is set to stop any motion once the drive exceeds this limit. This limit is based off of the feedback from the main LB382 sensors.

- Limit Switch 1 (Software only): When engaged by the trolley or carriage, this sensor indicates to the control system that it has been reached. 
- Limit Switch 2 (Software and hardware): When engaged by the trolley or carriage, this sensor indicates to the control system that it has been reached and it disables the high voltage contactors from the servo amplifiers and engages the $\mathrm{X}-\mathrm{Y}$ brakes independent of the tracker controller.

The trolley and carriage incorporate geometry designed such that once a limit switch is engaged, it remains engaged even if the system impacts the hard stop. It can only be disengaged by reversing direction and exiting the limit switch position. The purpose of the sequential limit switches is to assure that no high speed collisions into bump stops are possible. Even if the controller software does not respond appropriately to the first limit switch, the second limit switch will stop the drive by disabling power and engaging the brakes.

\subsection{Faults}

Fault monitoring is an important function for the tracker controller. The various fault conditions monitored are:

- Over-travel - Both software and limit switches continually monitor for over-travel conditions.

- Over-torque - Torque limiting couplings are used to mate the drive motors to the drive screws for all six X-Y motors. Proximity sensors monitor the state of these couplings. In the event of an over-torque condition the controller disables the high voltage contactors from the servo amplifiers and engages the brakes.

- $\underline{\text { Skew }}$ - Both software and skew limit switches continually monitor for excessive bridge skew conditions.

- Over-current - Software continually monitors the motor current. Due to the repetitive nature of the tracker motion, the current required is well defined. If the motor current radically diverges from this amount then a fault is generated.

- Over-temperature - Temperature sensors mounted on the motor housings are used to detect over-temperature conditions.

- Linear Sensors - The PT5DC absolute sensors are continually compared to the LB382 sensors. A fault is generated if the indicated position from the two sensors is not the same within a certain tolerance.

- Rho Sensors - The EQN437 absolute sensor is continually compared to the SIKO MSK1000. A fault is generated if the indicated position from the two sensors is not the same within a certain tolerance.

- Following Error - Excessive following error will generate a fault condition in the controller

- Over-Speed - Excessive carriage speed will generate a fault condition in the controller

- Load Cell - Out of range load cell outputs will generate a fault condition in the controller

A fault causes the controller to stop the tracker motion and engage the brakes. Faults such as the $2^{\text {nd }}$ travel limit switches and the skew limit switches will automatically disable the high voltage contactors and engage the brakes.

\section{DSPACE CONTROLLER}

\subsection{Modular Hardware}

A dSPACE modular controller was selected for the tracker. These controllers are widely used in the automotive and aerospace industries. This controller uses a single control board supplemented by several input/output boards. The modular hardware in the dSPACE controller is as follows:

- DS1005 - (1) PowerPC based $1 \mathrm{GHz}$ processor

- DS4504 ETX - (2) $100 \mathrm{Mbit} / \mathrm{s}$ Ethernet interface board

- DS3002 - (1) 6 channel incremental encoder interface board

- DS2002 - (1) 32 channel A/D board

- DS4003 - (2) 96 channel digital I/O board 
- PROFIBUS - (1) PROFIBUS master board

- DS5202 EnDat - (1) 8 channel EnDat 2.2 interface board

This equipment is housed in a PX20 rack mount enclosure located in the electrical control room.

\section{$5.2 \mathrm{I} / \mathrm{O}$}

The various communication boards in the dSPACE controller interface with different systems on the tracker:

- DS4504 ETX - Two Ethernet boards are installed in the dSPACE controller. One is dedicated to communicate to the tracker DAS (data acquisition system) mounted on the top of the telescope. The DAS system on the tracker collects analog information and transmits it serially to the controller via an Ethernet link. The second Ethernet board communicates with the TCS (Telescope Control System). Tracker commands and feedback are sent over this Ethernet line. The second Ethernet link will also be used to send tracker logging data to the Telescope logging system.

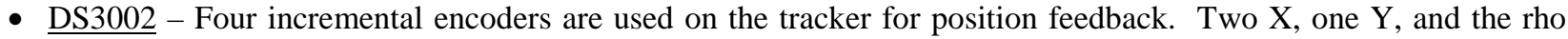
stage sensor. All four sensors are read by the DS3002 and are interpolated 4096 times.

- DS2002 - Direct analog measurements are necessary in the electrical room where the tracker controller is located. Various voltages and currents are measured and read into the 16-bit A/D board.

- DS4003 - Two digital I/O boards are installed in the dSPACE controller. The first is dedicated to the discrete sensors mounted on the tracker. These sensors, mainly limit switches and proximity sensors, feed through the DAS system down to the controller. The second DS4003 board is dedicated to the discrete sensors in the electrical room.

- PROFIBUS - Fourteen motor drives are required to run the tracker. These drives all contain PROFIBUS communication cards. Each drive is uniquely addressed and setup as a slave node. The dSPACE PROFIBUS card acts as the master on the network. dSPACE sequentially cycles through all of the drives to send new motor commands and receive drive status information.

- DS5202 EnDat - Seven EnDat 2.2 sensors are used on the tracker for position feedback. Each hexapod actuator contains one and the secondary sensor on the rho stage has one. This custom board directly interfaces with Heidenhain sensors to continually read position.

\subsection{Software}

The dSPACE controller is programmed using Matlab/Simulink. Control code is written and integrated with dSPACE tie-in blocks. The code is compiled using the Matlab 'Real-Time-Workshop' toolbox with a target of the dSPACE DS1005 control board. Once downloaded onto the controller, the processor runs at the desired real time frequency. The HETDEX tracker controller will run at $1000 \mathrm{~Hz}$. An engineering interface is built using dSPACE's ControlDesk software. This interface will have access to all of the variables and parameters within the tracker controller. The advantages of the dSPACE system with Matlab/Simulink controller are:

- Many input/output modular options available

- Coding is done at a high level which and can be completed by a range of technical staff members

- Updating the controller is simple and can be rebuilt and downloaded in minutes

\section{CONTROL ALGORITHM}

The control algorithm for the tracker has several parallel functions. These include motion control, compensation tables, mode arbitration, and fault detection and response.

\subsection{Motion Control}

$\underline{\mathrm{X} \text {-Drive }}$ 
The X-drives are mechanically independent from each other and offer difficulties in creating smooth concerted motion. A few issues which arise are:

- Different rail sag

- Different roller screw accuracy

- Different drive loads and friction

The control approach used relies on three elements:

1. Independent PID motion control - Each X-drive has a motion controller which bases its feedback off the LB382 sensor. Using a PID controller, the primary command for the drive is calculated.

2. Relative skew PID motion control - The difference between the upper and lower LB382 sensors produces a skew error. Using a separate PID controller, this error generates a supplementary command for each drive. The lagging drive receives a command to increase force while the leading drive is commanded to decrease force. The net force on the system remains the same and is dictated by the two independent controllers.

3. Compensation tables - Minor corrections are automatically implemented to account for the inaccuracies in the rails, roller screws, and other mechanical components.

Y-Drive / Constant Force Drive

The Y-axis system on the HETDEX tracker uses two drive systems operating in parallel to move the payload. These two systems have different purposes and operating methods:

- $\quad$ Y-Drive - The roller screw system controls the position

- $\quad$ Constant Force Drive (CFD) - The wire rope and drum system applies a constant offset force

The Y-Drive controls the position of the carriage based off the feedback from an LC183 sensor from Heidenhain corporation. A secondary string potentiometer sensor from Celesco, PT5DC, is used for start-up control and fault monitoring. A load cell reads the cable tension for the CFD. Using this load feedback, the CFD maintains a given force set point on the carriage at all times. Although both systems interact with the same load, the carriage, they have independent controllers. The Y-Drive controller is also responsible for moving the other axes on the tracker in addition to all fault monitoring and TCS communication. The CFD controller is a stand-alone solid-state controller.

Although the controllers for the Y-Drive and the CFD are independent they are connected via an interlock system. The purpose of this interlock is to assure that both systems are operational before Y-axis motion may commence. CFD enabling involves two steps:

1. Apply Power

2. Ready to Operate (RTO) signal is the enable command

This assures that the cable is tensioned as soon as the drive receives power. Either Y-drive requires the following steps to enable:

1. Apply Power

2. Five parallel signals must be in the 'good' condition to send an enable
a. CFD RTO signal is good
b. Y slew RTO signal is good
c. Y track RTO signal is good
d. Y-drive controller enable
e. Carriage Over-speed check

The three servo-drives share a common power contactor. Therefore when power is applied, all three drives are powered in parallel. 


\section{Hexapod-Drive}

Each hexapod actuator is individually controlled using a PID loop and a feedforward force command. The PID loop maintains the precise motion control while the feedforward command compensates for the near constant gravity load on the actuator. Each actuator will have a different offset loading due to its position in the hexapod. These offsets will be calibrated during initial testing.

Motion control commands will be in an X-Y-Z-rho-theta-phi format. These values will be converted to individual actuator leg lengths using a real time mathematic model of the hexapod.

Due to the leg length measurement technique, measuring screw rotations, and design of the hexapod actuators, a correction is necessary. The rod is not restrained about its own axis so it will rotate as the hexapod tips and tilts. This rotation causes a change in leg length which will not be measured by the rotary sensor. Correction for this length will be performed in software using the mathematical model for the hexapod.

Rho Drive

The Rho drive is controlled using a PID loop and a feedforward force command. The PID loop maintains the precise motion control while the feedforward command compensates for the eccentric mass on the rho stage. This eccentric mass load will be calibrated in initial testing to determine appropriate feedforward commands.

Motion commands will be simple rho positions. Using the MSK1000 sensor as position feedback, the controller will command motion.

\subsection{Compensation Tables}

Numerous compensation tables are necessary to build the appropriate mount model for the tracker. These tables can be broken down into several groups:

- X compensation

o Rail sag

o Roller screw accuracy

o Sensor accuracy

- Y compensation

o Rail sag

o Roller screw accuracy

o Sensor accuracy

- Hexapod compensation

o Actuator deflection

o Roller screw accuracy

o Sensor accuracy

o Carriage deflection

- Rho compensation

o Drum deflection

o Sensor accuracy

- Other 


\section{o Temperature \\ o PFIP deflection}

Tables will be generated and calibrated originally at the CEM (Center for Electromechanics) test installation but also at the final HETDEX installation. Some tables will remain somewhat static (e.g. Rail sag) while others may need occasional updating (e.g. roller screw accuracy).

\subsection{Mode Arbitration}

The controller is responsible for keeping track of which system is in 'control' of the tracker. This arbitration software decides which system can send legitimate commands and move the tracker. More information on this functionality is presented in Section 8.

\subsection{Fault Detection}

The controller continuously evaluates all fault conditions. If at any time it detects a fault, it will respond based on the severity of the fault. More information on this functionality is presented in Section 4.

\section{SIMULATIONS}

A primary advantage of the dSPACE controller is its close linkage with the MATLAB and Simulink computational and simulation environment. Exploiting this environment, a detailed integrated simulation of the complete tracker motion system, including the $\mathrm{X}$ drives/actuators, $\mathrm{Y}$ drives/actuators, hexapod actuators, rho stage actuators, and axis control algorithms was developed. Motion was modeled as ridged bodies within Simulink using the SimMechanics tool, with all appropriate degrees of freedom, constraints, and mass properties (from component solid models). The top level model in this simulation environment is shown in figure 3 , below.

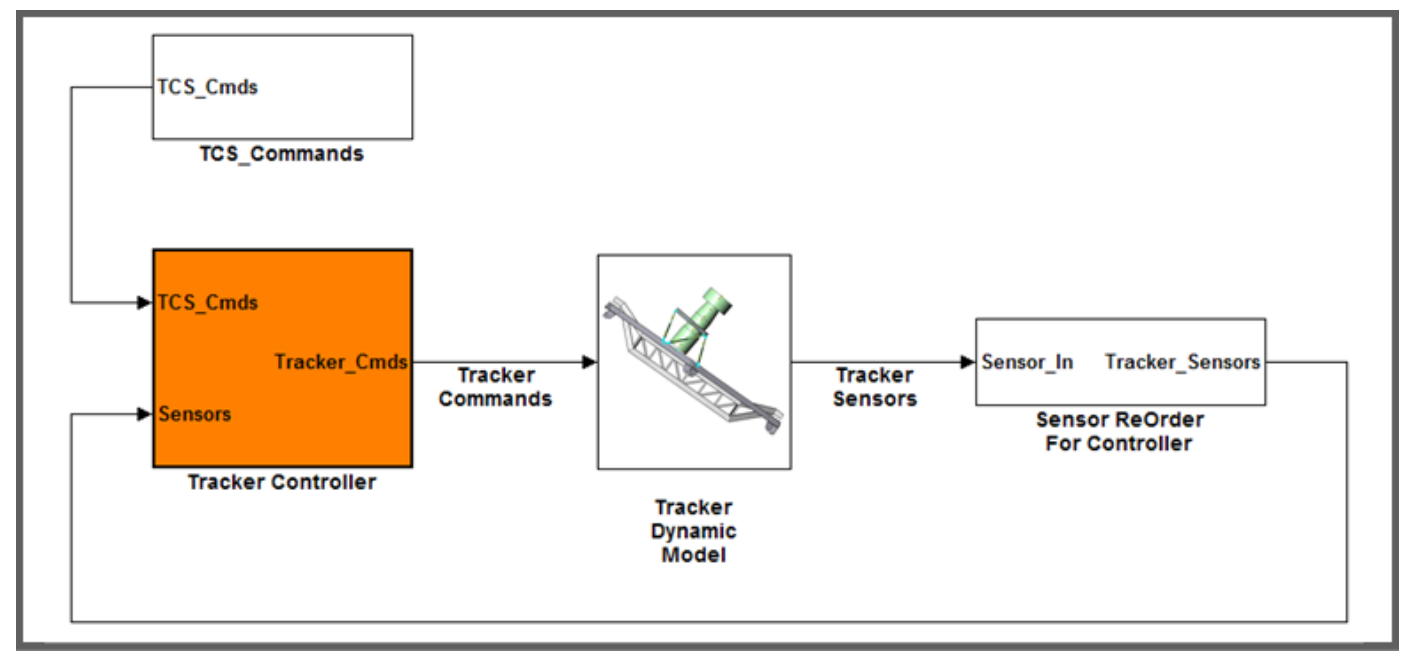

Figure 3. Simulink model of the tracker system. The "Tracker Dynamic Model” was completed using the SimMechanics tool. The rest of the simulation was based on normal Simulink blocks and S-functions.

Within the simulation model, modules were developed to represent sensor resolution, current resolution of the motor drive servo-amplifiers, and actuator friction characteristics. In the simulation model, all tracker control algorithms, including sensor filters, friction compensation, PID loops, and feed-forward algorithms are contained in the "Tracker Controller" block. These algorithms are built using the MATLAB GUI or built as S-functions using the MATLAB language (based on the MAPLE kernel). This Tracker Controller block then represents a stand-alone block that can be extracted (cut and paste) from the simulation environment and directly integrated into the dSPACE controller using MATLAB's realtime workshop. The realtime workshop is an auto-code generation process to generate ANSI C code so 
control algorithms developed in the simulation environment transfer directly to actual tracker controller hardware without re-programming. This process allows full use of MATLAB's powerful control development environment with very quick transition to control hardware.

For the model shown in Figure 3, the block labeled "TCS_Commands" represents the Tracker Controller link to the HET Telescope Control System. Simplified generation of tracker trajectories are developed in this block to exercise the model, debug and test the Tracker Controller, and tune the Tracker Controller. Outputs from the Tracker Controller are Tracker Commands to various tracker actuators within the Tracker Dynamic Model. As shown in Figure 3, simulated sensor output from the Tracker Dynamic Model are re-ordered and fed back to the Tracker Controller.

Prior to transferring the MATLAB based control system to the dSPACE hardware, extensive simulated tracking and slewing commands were exercised. Output from these simulations directly guided specifications for all tracker actuation subsystems. Model validation was completed as much as possible by correlating results within CEM engineering models in SolidWorks using SolidWorks specialized motion and constraint tools. Further model and control algorithm validation is ongoing on dedicated test rigs to characterize individual actuators or subsystems (e.g., a standalone single hexapod actuator test rig and a complete hexapod system test rig). CEM has extensive experience with developing high performance controlled motion systems and has repeatedly benefitted from the simulation based design process enabled by the MATLAB-dSPACE integrated system described in this section.

\section{CONTROL MODES}

Multiple control modes are necessary to satisfy the requirements for the HETDEX tracker. The tracker controller can operate using the following three modes:

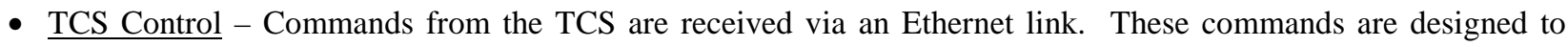
perform all science and general move requirements. This is the default operating mode.

- Tracker Control - Commands are generated by the tracker controller itself. Interfacing the system is done via the Tracker Engineering Interface. This interface is useful for debugging and maintenance.

- Manual Pendant Control - The manual pendant system is a small hand held controller with several momentary style buttons. These buttons send 'jog' commands to the tracker controller. This interface is useful for maintenance.

Switching between each control mode requires an arbitration system to assure that only one system can send legitimate commands. This arbitration is performed by a subroutine in the tracker controller. The guiding functions for control mode arbitration are:

- The system in control retains control until it relinquishes control

- A system must request control from the arbitration software

- Commands from a system which is not in control are ignored

\section{CONCLUSIONS}

Controller development on the HETDEX high precision tracker controller is proceeding. The suite of sensors required for control has been specified and is in procurement. Controller software is being developed in the Matlab/Simulink simulation environment and then tested incrementally on test stands in the laboratory. Compensation tables will be designed and calibrated using the tracker laboratory test stand and then recalibrated once the tracker is installed at HET. Major subsystem testing will commence this summer and fall. Development will continue through testing at CEM through early 2011. 


\section{ACKNOWLEDGEMENT}

HETDEX is led by the University of Texas at Austin McDonald Observatory and Department of Astronomy with participation from the Universitäts-Sternwarte of the Ludwig-Maximilians-Universität München, the Max-PlanckInstitut für Extraterrestriche-Physik (MPE), Astrophysikalisches Institut Potsdam (AIP), Texas A\&M University, Pennsylvania State University, and the HET consortium. In addition to Institutional support, HETDEX is funded in part by gifts from Harold C. Simmons, Robert and Annie Graham, The Cynthia and George Mitchell Foundation, Louis and Julia Beecherl, Jim and Charlotte Finley, Bill and Bettye Nowlin, Robert and Fallon Vaughn, Eric Stumberg, and many others, by AFRL under agreement number FA9451-04-2-0355, and by the Texas Norman Hackerman Advanced

Research Program under grants 003658-0005-2006 and 003658-0295-2007.

\section{REFERENCES}

[1] Ramsey, L.W., et al., 1998, “The early performance and present status of the Hobby-Eberly Telescope,” Proc. SPIE 3352, p.34

[2] Hill, G. J., Gebhardt, K., Komatsu, E., Drory, N., MacQueen, P. J., Adams, J. J., Blanc, G. A., Koehler, R., Rafal, M., Roth, M. M., Kelz, A., Gronwall, C., Ciardullo, R., Schneider, D. P., "The Hobby-Eberly Telescope Dark Energy Experiment (HETDEX): Description and Early Pilot Survey Results," ASP Conf. Series, 115-118 (2008)

[3] Savage, R.D., et al., "Current status of the Hobby-Eberly Telescope wide-field upgrade", Proc. SPIE 7733-149 (2010)

[4] Booth, J. A., Ray, F. B., and Porter, D. S., "Development of a star tracker for the Hobby Eberly Telescope", Proc. SPIE 3351, 20 (1998)

[5] Burge, J. H. et al, "Development of a wide-field spherical aberration corrector for the Hobby-Eberly Telescope", Proc. SPIE 7733-51 (2010)

[6] Hill, G. J., MacQueen, P. J., Palunas, P., Shetrone, M. D., Booth, J. A., "Present and Future Instrumentation for the Hobby-Eberly,” Proc. SPIE, 6269, 626907 (2006)

[7] Kelz, A., Roth, M. M., Bauer, S. M., Padilla, Y., Popow, E., Hill, G. J., Vattiat, B. L., Good, J. M., Murphy, J. D., Savage, R. D., Soukup, I. M., Mollison, N. T., "Production and performance of replicable integral field units for VIRUS”, Proc. SPIE 7735, 178 (2010)

[8] Hill, G. J., Adams, J. J., Blanc, G., Booth, J. A., Chonis, T. S., Cornell, M. E., DePoy, D. L., Drory, N., Gebhardt, K., Good, J. M., Grupp, F. U., Kelz, A., Lee, H., Marshall, J. L., MacQueen, P. J., Mollison, N. T., Murphy, J. D., Rafal, M. D., "VIRUS: a massively replicated 33k fiber integral field spectrograph for the upgraded Hobby-Eberly Telescope”, Proc. SPIE 7735, 21 (2010)

[9] Worthington, M. S., Nichols, S. P., Good, J. M., Zierer, J. J., Mollison, N. T., Soukup, I. M., "Design and analysis of the Hobby-Eberly Telescope dark energy experiment (HETDEX) bridge”, Proc. SPIE 7733, 147 (2010)

[10] Mollison, N. T., Mock, J. R. Soukup, I. M., Beets, T. A., Good, J. M., Beno, J. H., Kriel, H. J., Hinze, S. E., Wardell, D. R., "Design and development of a long-travel positioning actuator and tandem constant force actuator safety system for the Hobby-Eberly Telescope wide-field upgrade", Proc. SPIE 7733, 150 (2010)

[11]Zierer, J. Z., Mock, J. R., Beno, J. H., Lazzarini, P. G., Fumi, P., Anaclerio, V., Good, J. M., "The development of high-precision hexapod actuators for the Hobby-Eberly Telescope Dark Energy Experiment (HETDEX)", Proc. SPIE 7733, 49 (2010)

[12] G.A. Wedeking, et al., "Kinematic optimization of upgrade to the Hobby-Eberly Telescope through novel use of commercially available three-dimensional CAD package,” Proc. SPIE, 7733-148 (2010) 